\title{
Tyrosinase Inhibitory Effects and Antioxidant Properties of Paeonol and Its Analogues
}

\author{
Tian-Hua ZHU, Yan-Ying Yu ${ }^{*}$ and Shu-Wen CAO
}

Department of Chemistry, Nanchang University, Nanchang 330031, PR China.

Received December 19, 2012; Accepted March 4, 2013

With the aim to find out structural features for the tyrosinase inhibitory activity, the inhibitory effects of seven paeonol analogues on the diphenolase of mushroom tyrosinase, the interaction between the inhibitors and the copper ions, and the antioxidant activity by DPPH radical were investigated. These paeonol analogues had suggested remarkable inhibition toward tyrosinase. Among them, paeonol (a) exhibited the strongest inhibition activity $\left(\mathrm{IC}_{50}=0.21 \mathrm{mM}\right)$ as well as showed potent scavenging activity on the DPPH radical. The inhibition kinetics revealed that paeonol (a) was a mixed-type inhibitor, $2^{\prime}$-hydroxy acetophenone (c), 4'-hydroxy acetophenone (d), and 2,4'-dihydroxy acetophenone (e) were competitive inhibitors, while acetophenone (b), 2'-methoxyacetophenone (f), and 4'-methoxy acetophenone (g) behaved as noncompetitive inhibitors. The maximum absorbing wavelengths of compounds (c), (d), and (e) showed different significant blue shifts, which could explain that they exhibited competitive inhibition by forming a chelate with the copper ions at the catalytic domain of the tyrosinase.

Keywords: paeonol, acetophenone, tyrosinase inhibitor, DPPH radical, copper ions chelation

\section{Introduction}

Tyrosinase (EC 1.14.18.1), known as polyphenol oxidase, is a multifunctional copper-containing metalloenzyme widely distributed in nature. It mainly catalyzes the $o$-hydroxylation of monophenols to the corresponding catechols, and the oxidation of monophenols to the corresponding $o$-quinones (Yoon et al., 2009). Consequently, a series of high reactive quinones are produced to initiate the pigmentation and excessive activation of tyrosinase can cause various dermatological disorders, such as Parkinson and other degenerative diseases (Asanuma et al., 2003).

One of the reactions catalyzed by the tyrosinase is the oxidation of L-dopa to L-dopaquinone by utilising molecular oxygen. In addition, reactive oxygen species (ROS) and free radical-mediated reactions are associated with various diseases, such as diabetes mellitus, cancer, ageing, skin disorders, and neurodegenerative diseases (Pham-Huy et al., 2008). If the inhibitors we have designed can weaken the tyrosinase activity as well as scavenge ROS effectively, the production of melanin and the damage of ROS to our human beings will be decreased to a great extent. Hence, tyrosinase inhibitors have become increasingly important in cosmetic

*To whom correspondence should be addressed.

E-mail: yuyanying@ncu.edu.cn
(Guevara and Pandya, 2001) and medicinal (Kanost et al., 2004) industry, the food industry (Lunadei et al., 2011) and agriculture (Guerrero and Rosell, 2005) to prevent hyperpigmentation.

In recent years, a large number of naturally occurring and synthetic tyrosinase inhibitors have been reported. There are many tyrosinase inhibitors, such as hydroquinone (Garcia and Fulton Jr, 1996), ascorbic acid (Kameyama et al., 1996), arbutin (Nakajima et al., 1998), kojic acid (Mishima et al., 1994), aromatic aldehydes (Jimenez et al., 2001; Chen et al., 2003), aromatic acids (Chen et al., 2005; Si et al., 2011), aromatic alcohol (Liu et al., 2007; Zhu et al., 2011), and tropolone (Valero et al., 1991). It has been suggested that the addition of a methoxy group at the meta or para position of salicylaldehyde shows different inhibition strength. Alkoxybenzoic acids have been studied for the inhibition of mushroom tyrosinase (Huang et al., 2006), revealing that different alkoxy substitutions induce various inhibition type (Chen et al., 2005). The inhibition of 2 '-phenylethanol, $2^{\prime}$-phenylacetaldehyde and 2'-phenylacetic acid on the activity of mushroom tyrosinase indicating that the functional group on the benzene ring group played an important role in the enzyme inhibition (Zhu et al., 2011).

Paeonol (structure was shown in Fig. 1(a)), belonging to aromatic ketone, one of the primary bioactive components 


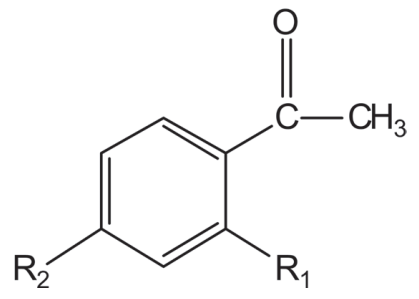
(a): $\mathrm{R} 1=\mathrm{OH}, \mathrm{R} 2=\mathrm{OCH} 3$;
(b): $\mathrm{R} 1=\mathrm{H}, \mathrm{R} 2=\mathrm{H}$;
(c): $\mathrm{R} 1=\mathrm{OH}, \mathrm{R} 2=\mathrm{H}$;
(d): R1 = H, R2 = OH;
(e): $\mathrm{R} 1=\mathrm{OH}, \mathrm{R} 2=\mathrm{OH}$;
(g): R1 = H, R2 = OCH3;
(f): $\mathrm{R} 1=\mathrm{OCH} 3, \mathrm{R} 2=\mathrm{H}$;

Fig. 1. Chemical structures of paeonol and its analogues.

isolated from Chinese national flower-peony, is known to have a series of pharmaceutical properties including antiinflammatory, anti-oxidant and also known as traditional skin-whitening agent, a potential tyrosinase inhibitor (Gong et al., 2006). However, the inhibitory effects of other aromatic ketones on the enzymatic activity of mushroom tyrosinase are little explored. It has been confirmed that ortho- and para- substitutes of benzene ring produce bigger influence on mushroom tyrosinase for the oxidation of L-DOPA (Liu et al., 2007). Therefore, the aim of the present paper is, to study the inhibitory kinetics of paeonol analogues which all are 2,4 substituted acetophenones on the enzyme activity and evaluate the kinetic parameters and the inhibition mechanisms, to investigate the interaction between the inhibitors and the copper ions, and to measure the antioxidant activity of paeonol analogues and finally come up with the structureactivity relationship. We hope they can be used to provide the basis for developing novel effective tyrosinase inhibitors and searching for new whitening agents in cosmetic preparations or antibrowning agents for food products.

\section{Materials and Methods}

Materials Mushroom tyrosinase (EC 1.14.18.1), L-3,4dihydroxyphenylalanine (L-DOPA), and 2,2-diphenyl-1picrylhydrazyl (DPPH), dimethyl sulfoxide (DMSO) were purchased from Sigma Chemical Co (St. Louis, MO, USA). The specific activity of the enzyme was $1881 \mathrm{U} / \mathrm{mg}$. Paeonol (a) (see Fig. 1 for structures), acetophenone (b), 2'-hydroxy acetophenone (c), 4'-hydroxy acetophenone (d), 2,4'-dihydroxy acetophenone (e), 2'-methoxyacetophenone (f), and 4'-methoxy acetophenone (g) were obtained from J \& K Chemical Co. (Shanghai, China). All other reagents were of analytical grade. The water used was re-distilled and ionfree. All the datas were recorded using Shimadzu UV-2450 spectrophotometer (Japan).

Tyrosinase inhibitory activity The assay of the enzyme activity was performed as previously described by Xie et al. (2003) with minor modification. $0.1 \mathrm{~mL}$ of different concentrations of effector (dissolved in DMSO solution), was added to $2.8 \mathrm{~mL}$ L-DOPA in $\mathrm{Na}_{2} \mathrm{HPO}_{4}-\mathrm{NaH}_{2} \mathrm{PO}_{4}$ buffer (50 mM, pH 6.8), and then $0.1 \mathrm{~mL}$ of aqueous solution of mushroom tyrosinase was added to the assay mixture with a total volume of $3 \mathrm{~mL}$. The final concentration of mushroom tyrosinase was $3.75 \mu \mathrm{g} / \mathrm{mL}$. The solution was immediately monitored by measuring the dopachrome formation at 475 $\mathrm{nm}\left(\varepsilon=3700 \mathrm{M}^{-1} \mathrm{~cm}^{-1}\right)$ for $5 \mathrm{~min}$ using a Shimadzu UV2450 spectrophotometer. The reaction was carried out under a constant temperature of $30^{\circ} \mathrm{C}$. The extent of inhibition by the addition of the sample was expressed as the percentage necessary for $50 \%$ inhibition $\left(\mathrm{IC}_{50}\right)$. Controls, without inhibitor but containing 3.3\% DMSO.

Determination of the inhibition type and inhibition constants The inhibition type was determined by LineweaverBurk plots, and the inhibition constants were obtained by the second plots of the apparent $\mathrm{Km} / \mathrm{Vmapp}$ or 1/Vmapp versus the concentration of the inhibitor (Chen et al., 2002).

Copper ions interaction study To investigate the interaction between the copper ions and the above substances, stock solutions of compounds (a) - (g) (1 mM) were prepared in methanol. Then $25 \mu \mathrm{M}$ solutions were prepared in a cuvette containing phosphate buffer ( $50 \mathrm{mM}, \mathrm{pH} 6.8)$, and the absorption spectra were recorded between 200 and 600 nm. Scans with $10,25,50$, or $100 \mu \mathrm{M} \mathrm{CuSO}_{4}$ were taken after 10 s using a UV-2450 spectrophotometer, which were compared to the substances alone.

Radical-scavenging activity assay The radical-scavenging activities of the samples on DPPH were determined according to the modified method described previously by Lee et al. (2008). First, $1.0 \mathrm{~mL}$ of $1 \mathrm{mM}$ different sample solutions (dissolved in ethanol), and $0.5 \mathrm{~mL}$ of ethanolic solution of $0.6 \mathrm{mM}$ DPPH were put into the test tube. Then, 3.5 $\mathrm{mL}$ of ethanol was added to get a $5 \mathrm{~mL}$ system. $1.0 \mathrm{~mL}$ of ethanol was added to the tube in place of scavengers as the control. The decrease in absorbance of DPPH at $517 \mathrm{~nm}$ after $30 \mathrm{~min}$ at room temperature was measured using a UV-2450 spectrophotometer. The inhibition (\%) of DPPH absorbance was calculated based on the expression $\left(A_{\text {control }}-A_{\text {test }}\right) \times 100 /$ $A_{\text {control }}$, where $A_{\text {control }}$ is the absorbance of the control (DPPH solution in the absence of a test sample) and $\mathrm{A}_{\text {test }}$ is the absorbance of the test sample.

\section{Results and Discussion}

Effects of compounds (a) - (g) on the diphenolase activity of mushroom tyrosinase The inhibition of compounds (a), (b), (c), (d), (e), (f), and (g) on the oxidation of L-DOPA by mushroom tyrosinase was determined, as shown in Fig. 2 , indicating paeonol and its analogues inhibited tyrosinase in a dose-dependent manner. The $\mathrm{IC}_{50}$ values were listed in 

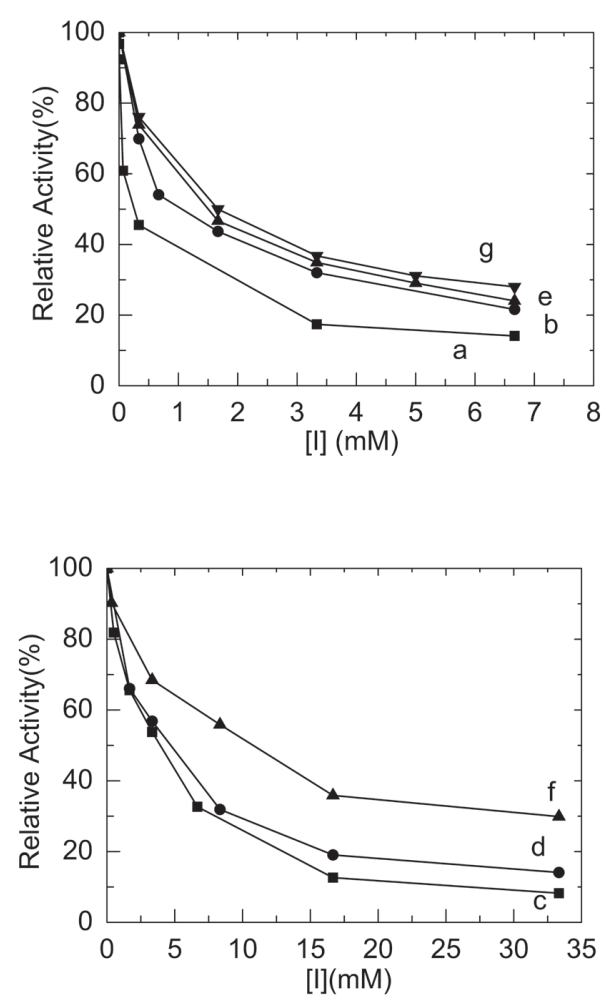

Fig. 2. Dose-dependent inhibitory effects of paeonol analogues on the diphenolase activity of mushroom tyrosinase.

Table 1. Inhibitory effects and constants of the paeonol analogues on mushroom tyrosinase

\begin{tabular}{cclcc}
\hline \multirow{2}{*}{ Compd } & $\begin{array}{c}\mathrm{IC}_{50} \\
(\mathrm{mM})\end{array}$ & Inhibition type & \multicolumn{2}{c}{$\begin{array}{c}\text { Inhibition constants } \\
(\mathrm{mM})\end{array}$} \\
\cline { 4 - 5 } & & & $\mathrm{KI}$ & $\mathrm{KIS}$ \\
\hline $\mathrm{a}$ & 0.207 & mixed & 0.201 & 1.447 \\
$\mathrm{~b}$ & 0.916 & non-competitive & 0.686 & 0.686 \\
$\mathrm{c}$ & 3.096 & competitive & 1.574 & \\
$\mathrm{~d}$ & 3.820 & competitive & 2.265 & \\
$\mathrm{e}$ & 1.248 & competitive & 0.826 & \\
$\mathrm{f}$ & 9.278 & non-competitive & 6.358 & 6.358 \\
$\mathrm{~g}$ & 1.674 & non-competitive & 0.994 & 0.994 \\
\hline
\end{tabular}

Table 1 for comparison. Among them, compound (a) had the most potent inhibition activity of mushroom tyrosinase with the $\mathrm{IC}_{50}$ value of $0.21 \mathrm{mM}$, and the inhibitory strength of $p$ methoxy acetophenone was about 6 times stronger than that of $o$-methoxy acetophenone.

It can be speculated that acetophenone exhibited tyrosinase inhibitory effect due to the formation of a Schiff base with the primary amino group of tyrosinase as aromatic aldehydes. Compound (a) showed the strongest inhibitory effect due to the introduction of an electron donor methoxy group with high hydrophobicity at the para- position stabilizing the Schiff base and the ortho hydroxyl group forming a quasi

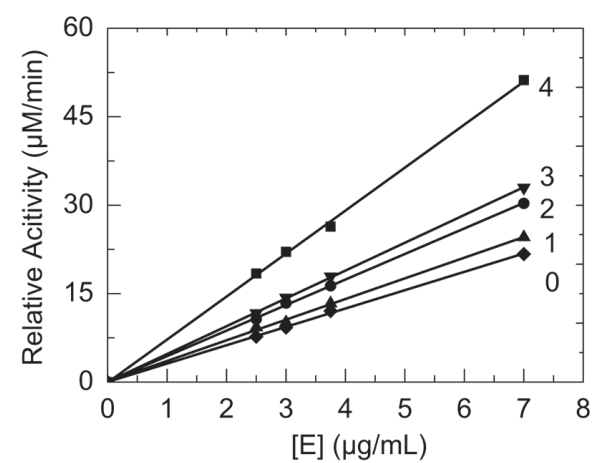

Fig. 3. Effects of concentrations of tyrosinase on its activity for the catalysis of L-DOPA at different concentrations of paeonol (a). Concentrations of paeonol for curves $0-4$ were $0,0.05,0.1,0.2$, and $0.4 \mathrm{mM}$.

six-member ring through intramolecular hydrogen bonding to produce a more stable structure (Chen et al., 2003). It has been suggested that the more stable the Schiff base is, the more potent the inhibitory activity. The more hydrophobic the electron donor group at the para position, the stronger the inhibition. Furthermore, the hydroxyl group of paeonol is able to chelate copper ions in the active site of tyrosinase. Therefore, replacements of $o$-hydroxy and $p$-methoxy moieties of acetophenone are more appreciable for the activity enhancement. Phenolic compounds (c), (d), and (e) did not serve as substrates, they cannot be oxidized by tyrosinase.

The inhibition mechanism of compounds (a) - (g) against the activity of mushroom tyrosinase The inhibition mechanism of compound (a) on mushroom tyrosinase for the oxidation of L-DOPA was shown in Fig. 3. The results showed straight lines passing through the origin, which indicate a reversible inhibition, the other inhibitors $(b)-(g)$ showed the same behavior. Tyrosinase activity was inhibited, but the amount of efficient enzyme was not decreased in the presence of these inhibitors. The results showed that the catalytic rate did not make any change during the variation of the concentration. The inhibitors appeared to bind tyrosinase very quickly and did not induce further conformational change in the protein.

The inhibition pattern and constants of compounds (a) (g) on the activity of mushroom tyrosinase The inhibition kinetics of the enzyme for oxidation of L-DOPA by selected compounds (a), (e), and (b) were illustrated in Fig. 4-6, respectively. The Lineweaver-Burk plots in Fig. 4 gave a family of lines intersected in the second quadrant, indicating that (a) was a mixed-type inhibitor. The constant for inhibitor binding with the free enzyme $\left(\mathrm{K}_{\mathrm{I}}\right)$ was obtained from a plot of the slopes of the straight lines versus the inhibitor concentrations; the constant for inhibitor binding with the enzyme- 


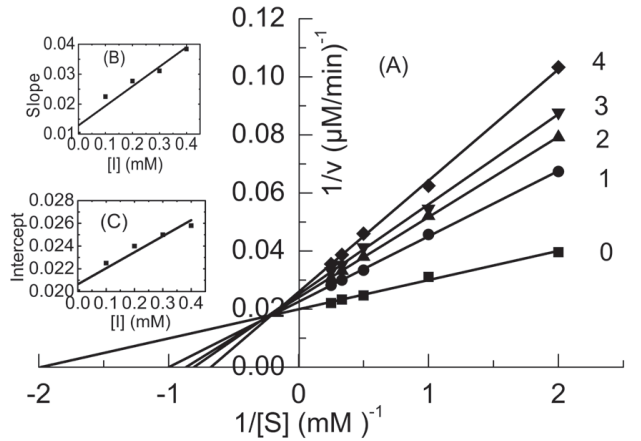

Fig. 4. Lineweaver-Burk plots of the inhibition by paeonol (a) of the mushroom tyrosinase mediated catalysis of L-DOPA (A). The inset represents the second plot of the intercept (B) and the slope (C) versus the concentrations of inhibitor to determine the inhibition constant $\mathrm{K}_{\mathrm{I}}$ and $\mathrm{K}_{\mathrm{IS}}$. The concentrations of paeonol (a) for curves 0 -4 were $0,0.1,0.2,0.3$ and $0.4 \mathrm{mM}$, respectively.

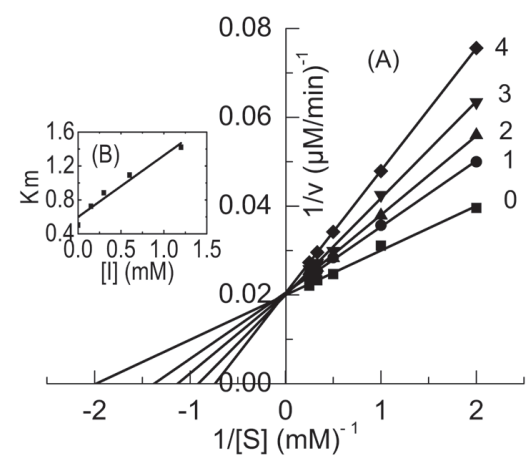

Fig. 5. Lineweaver-Burk plots of mushroom tyrosinase on the presence of 2,4'-dihydroxy acetophenone (e) during the oxidation of L-DOPA (A). The inset represents the second plot of the $\mathrm{K}_{\mathrm{m}}(\mathrm{B})$ versus the concentrations of inhibitor to determine the inhibition constant. The concentrations of 2,4'-dihydroxy acetophenone (e) for curves $0-4$ were $0,0.15,0.3,0.6$, and $1.2 \mathrm{mM}$, respectively.

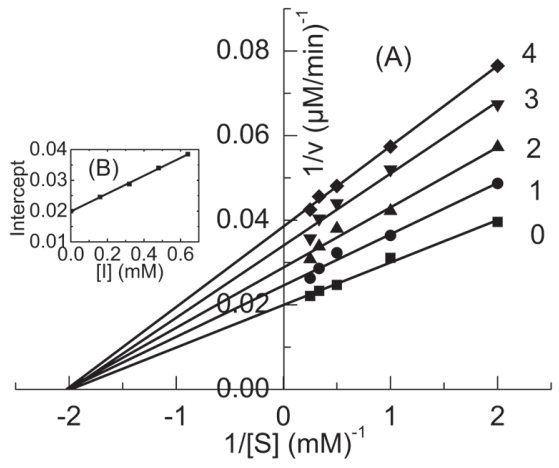

Fig. 6. Lineweaver-Burk plots of mushroom tyrosinase for inhibition of acetophenone (b) on the oxidation of L-DOPA (A). The inset represents the second plot of the Intercept (B) versus the concentrations of inhibitor to determine the inhibition constant. The concentrations of acetophenone (b) for curves $0-4$ were $0,0.16,0.32,0.48$, and $0.64 \mathrm{mM}$, respectively. substrate complex $\left(\mathrm{K}_{\mathrm{IS}}\right)$ was gained from a plot of the vertical intercepts versus the inhibitor concentrations, which are linearly fitted, showing that compound (a) has a single inhibition site or a single class of inhibition site on tyrosinase ( $\mathrm{Si}$ et al., 2011), as shown in the inset. The inhibition constants are summarized in Table 1 for comparison. Because $\mathrm{K}_{\mathrm{IS}}>\mathrm{K}_{\mathrm{I}}$, the affinity of paeonol with the free enzyme is greater than that of the inhibitor with the enzyme-substrate complex.

The inhibitory type of (c), (d), and (e) on the enzyme activity shared the same. Lineweaver-Burk plots of the inhibition kinetics of tyrosinase for selected compound (e) is shown in Fig. 5, as the concentration of compound (e) increased, the $\mathrm{K}_{\mathrm{m}}$ for tyrosinase increased in a dose-dependent manner without changing $\mathrm{V}_{\max }$, which indicates compound (e) as a competitive inhibitor of tyrosinase. The result showed that these inhibitors could only bind with free enzyme. The inhibition constant for the inhibitor $\left(\mathrm{K}_{\mathrm{I}}\right)$, was obtained from a plot of the intercept versus the inhibitor concentration, as shown in the inset.

Similarly, The inhibitory type of (b), (f), and (g) on the enzyme activity shared the same. The Lineweaver-Burk plots of compound (b) shown in Fig. 6, gave a family of straight lines, with different slopes but with a common intercept on the $1 /[\mathrm{s}]$ axis, indicating that they are non-competitive inhibitors. The behaviour observed showed that the inhibitor could combine with both the free enzyme and the enzymesubstrate complex. The inhibition constants $\left(\mathrm{K}_{\mathrm{I}}\right.$ and $\left.\mathrm{K}_{\mathrm{IS}}\right)$ can be obtained from a plot of the vertical intercept versus the inhibitor concentration as showed in Table 1.

It is evident to summarize that the above compounds with methoxy exhibited non-competitive inhibition type, showed competitive inhibition with mono- or dihydroxy, displayed mixed-type inhibition when both methoxy and hydroxyl existed.

The copper ions chelation ability Many substances have been reported to act as an tyrosinase inhibitor due to their innate function in chelating metal ions, maybe as a chelator to the copper ions in the tyrosinase (Kim et al., 2006; Khatib et al., 2005; Zhu et al., 2009). In order to explore and illustrate the underlying mechanism better, the direct interactions between the inhibitors and the copper ions were assessed routinely by UV-2450 spectrophotometer at $\mathrm{pH}$ 6.8. The effect of the increments of the $\mathrm{CuSO}_{4}$ concentration on the spectral characteristics of each compound is measured in terms of red shifts/blue, as a result of complex formation between the copper ions and the tested compounds. A solution of each compound (a) - (g) $(25 \mu \mathrm{M})$ in PBS buffer was scanned with $0,10,25,50$, or $100 \mu \mathrm{M} \mathrm{CuSO}_{4}$, and the spectra obtained were compared.

The characteristic maximum at $276.5 \mathrm{~nm}$ of compound 

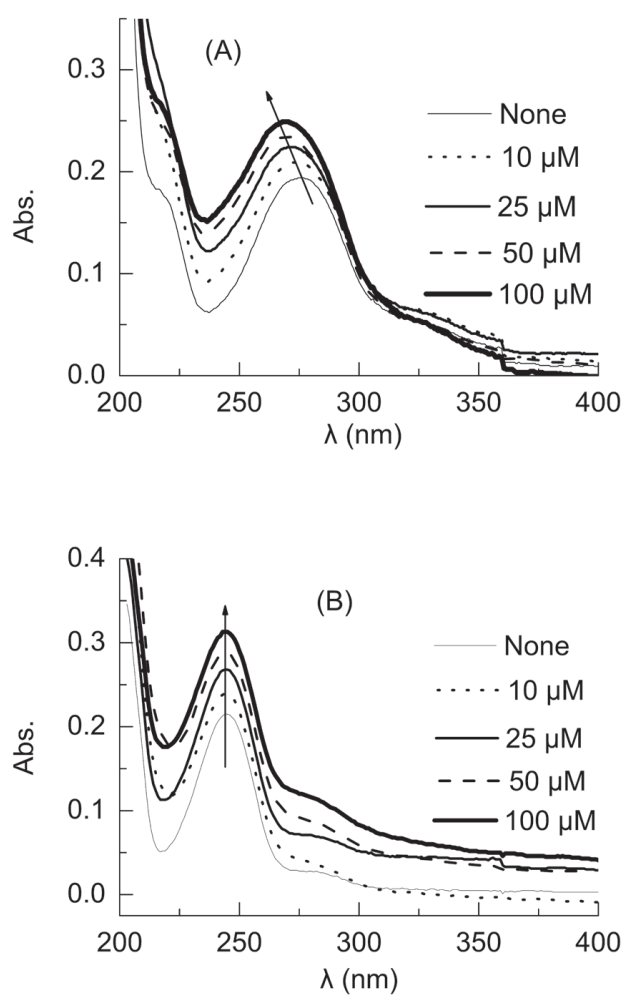

Fig. 7. Copper ions chelating ability. (A) and (B) are the UV-vis absorption spectra of 4-hydroxy acetophenone (d) and acetophenone (b) at phosphate buffer $(50 \mathrm{mM}, \mathrm{pH} 6.8)$ after the addition of different concentrations of copper ions, respectively.

(d) showed various significant blue shifts peaks with the addition of different concentrations of copper ions, shifting to $268.5 \mathrm{~nm}$ after added $100 \mu \mathrm{M} \mathrm{CuSO}_{4}$ (Fig. 7A). Similar results were obtained with compounds (a), (c), and (e), with the addition of copper ions, the maximum absorbing wavelengths $\lambda_{\max }$ were shifted from 311 to $308 \mathrm{~nm}$, from 322.5 to $316 \mathrm{~nm}$ and from 321.5 to $314 \mathrm{~nm}$, respcetively. However, it is obvious that the characteristic values of compounds (b), (f), and (g) were almost unchanged, as shown in Fig. 7B.

As such, it is concluded that the characteristic maximum absorbing wavelengths of hydroxy substituted acetophenone showed different significant blue shifts while that of methoxy substituted acetophenone didn't make any change. This could explain that the hydroxy compounds (c), (d), and (e) exhibited competitive inhibition by forming a chelate with the copper ions at the catalytic domain of the tyrosinase to prevent L-DOPA binding to the active sites of tyrosinase.

$D P P H$ radical-scavenging activities Tyrosinase is a kind of oxidase, one of the reactions catalyzed by the tyrosinase is the oxidation of L-dopa to L-dopaquinone by using molecular oxygen. The reducers which have strong radicalscavenging activity are all potent inhibitors of tyrosinase. The mechanism is that inhibitors cleared the peroxy radicals, terminated radical chain initiation, and weakened the role of

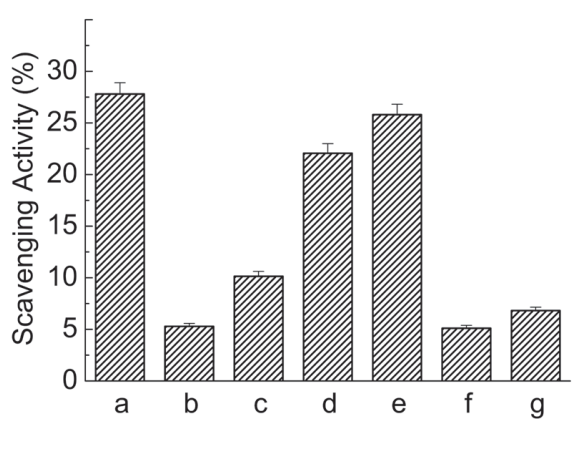

Fig. 8. DPPH radical-scavenging activities of compounds a-g with an identical final concentration of $200 \mu \mathrm{M}$.

oxygen of tyrosinase, which weakened the role of tyrosinase (Chen et al., 2012). Therefore, free radical scavenging compounds could also be used as a cosmetic ingredient to relieve skin aging (Tanaka et al., 2004).

The DPPH radical-scavenging assay was chosed to examine antioxidative properties of compounds (a) - (g). All the compounds were tested at $200 \mu \mathrm{M}$ final concentration. The results are shown in Fig. 8. In general, among these compounds, paeonol displayed the most potent inhibitory activities; (b), (f), and (g) which contain no hydroxyl group approximately showed no inhibitory activity; Monohydroxyl compounds (c) and (d) exhibited certain inhibitory activities, which was weaker than that of dihydroxyl compounds (e). In contrast to the result of tyrosinase, numbers and position of phenolic hydroxyl groups and methoxy substitutes in acetophenone showed major effect on their inhibitory activities. It has been confirmed that these substance not only acted as tyrosinase inhibitors but also provided antioxidant activity.

In this paper, the effects of paeonol and its analogues on the oxidation of L-DOPA by mushroom tyrosinase were studied. These analogues have been found having remarkable inhibition on mushroom tyrosinase and the inhibition was displayed as reversible. Despite the structural similarities, the inhibition mechanisms and inhibitory effects of the seven compounds are different from each other to some extent. Replacement of $o$-hydroxy and $p$-methoxy moieties of acetophenone is more favorable for the activity enhancement. The kind, the number, and the position of the functional group on the benzene ring group played an important role in the enzyme inhibition activity. The inhibition kinetic of these compounds revealed that the inhibition type was induced by the kind of substitutions at ortho or para position. Previous studies have recognized an apparently potent inhibitory effect of compounds with hydroxyl groups on tyrosinase (Yan et al., 2009; Yamazaki et al., 2009). The hydroxyl groups in compounds carry out an attack on the coppers of tyrosinase 
active site, competing with substrate L-DOPA in a complex manner (Munoz-Munoz et al., 2008). Copper ions chelation assay could be used to explain that the hydroxy compounds (c), (d), and (e) exhibited competitive inhibition by forming a chelate with the copper ions at the catalytic domain of the tyrosinase to inhibit the tyrosinase. In conclusion, these results suggest that they may serve as an potential agent for the prevention and treatment of pigmentation and the inhibition of melanogenesis.

Acknowledgments This work was supported by National Natural Sciences Foundation of China (NO. 20962014).

\section{References}

Asanuma, M., Miyazaki, I. and Ogawa, N. (2003). Dopamine- or L-DOPA-induced neurotoxicity: The role of dopamine quinone formation and tyrosinase in a model of Parkinson's disease. $J$. Neurotox. Res., 5, 165-176.

Chen, Q.X. and Kubo, I. (2002). Kinetics of mushroom tyrosinase inhibition by quercetin. J. Agric. Food Chem., 50, 4108-4112.

Chen, Q.X., Song, K.K., Wang, Q. and Huang, H. (2003). Inhibitory effects on mushroom tyrosinase by some alkylbenzaldehydes. $J$. Enzym. Inhib. Med. Chem., 18, 491-496.

Chen, Q.X., Song, K.K., Qiu, L., Liu, X.D., Huang, H. and Guo, H.Y. (2005). Inhibitory effects on mushroom tyrosinase by p-alkoxybenzoic acids. Food Chem., 91, 269-274.

Chen, L.H., Hu, Y.H., Song, W., Song, K.K., Liu, X., Jia, Y.L., Zhuang, J.X. and Chen, Q.X. (2012). Synthesis and Antityrosinase Mechanism of Benzaldehyde Thiosemicarbazones: Novel Tyrosinase Inhibitors. J. Agric. Food Chem., 60, 1542-15479.

Garcia, A. and Fulton Jr, J.E. (1996). The combination of glycolic acid and hydroquinone or kojic acid for the treatment of melasma and related conditions. Dermatol. Surg., 22, 443-447.

Gong, S.Z., Cheng J. and Yang Z.R. (2006). Inhibitory kinetics of paeonol on the activity of mushroom tyrosinase oxidizing LDopa. Acta Pharmaceutica Sinica., 41, 561-564.

Guerrero, A. and Rosell, G. (2005). Biorational approaches for insect control by enzymatic inhibition. Curr. Med. Chem., 12, 461469.

Guevara, I.L. and Pandya, A.G. (2001). Melasma treated with hydroquinone, tretinoin, and a fluorinated steroid. Int. J. Dermatol., 40, 212-215.

Huang, X.H., Chen, Q.X., Wang, Q., Song, K.K., Wang, J., Sha, L. and Guan, X. (2006). Inhibition of the activity of mushroom tyrosinase by alkylbenzoic acids. Food Chem., 94, 1-6.

Jimenez, M., Chazarra, S., Escribano, J., Cabanes, J. and Garc1'aCarmona, F. (2001). Competitive inhibition of mushroom tyrosinase by 4-substituted benzaldehydes. J. Agric. Food Chem., 49, 4060-4063.

Kameyama, K., Sakai, C., Kondoh, S., Yonemoto, K., Nishiyama,
S. and Tagawa, M. (1996). Inhibitory effect of magnesium Lascorbyl-2-phosphate (VC-PMG) on melanogenesis in vitro and in vivo. J. Am. Acad. Dermatol., 34, 29-33.

Kanost, M.R., Jiang, H. and Yu, X.Q. (2004). Innate immune responses of a lepidopteran insect, Manduca sexta. Immunol. Rev., 198, 97-105.

Khatib, S., Nerya, O., Musa, R., Shmuel, M., Tamir, D. and Vaya, J. (2005). Chalcones as potent tyrosinase inhibitors: the importance of a 2,4-substituted resorcinol moiety. J. Bioorg. Med. Chem., 13, 433-441.

Kim, D., Park, J., Kim, J., Han, C., Yoon, J. and Kim, N. (2006). Flavonoids as mushroom tyrosinase inhibitors: A fluorescence quenching study. J. Agric. Food Chem., 54, 935-941.

Lee, Y.L., Yang, J.H. and Mau, J.L. (2008). Antioxidant properties of water extracts from Monascus fermented soybeans. Food Chem., 106, 1127-1137.

Liu, S.H., Pan, I.H. and Chu, I.M. (2007). Inhibitory effect of phydroxybenzyl alcohol on tyrosinase activity and melanogenesis. Biol. Pharm. Bull., 30, 1135-1139.

Lunadei, L., Galleguillo, P., Diezma, B., Lleo, L. and Ruiz-Garcia, L. (2011). A multispectral vision system to evaluate enzymatic browning in fresh-cut apple slices. Postharvest Biol. Technol., 60, 225-234

Mishima, Y., Ohyama, Y., Shibata, T., Seto, H. and Hatae, S. (1994) Inhibitory action of Kojic acid on melanogenesis and its therapeutic effect for various human hyper-pigmentation disorders. Skin Res., 36, 134-150.

Munoz-Munoz, J.L., Garcia-Molina, F., Garcia-Ruiz, P.A., MolinaAlarcon, M., Tudela, J., Garcia-Canovas, F. and Rodriguez-Lopez, J.N. (2008). Phenolic substrates and suicide inactivation of tyrosinase: kinetics and mechanism. Biochem. J., 416, 431-440.

Nakajima, M., Shinoda, I., Fukuwatari, Y. and Hayasawa, H. (1998). Arbutin increases the pigmentation of cultured human melanocytes through mechanisms other than the induction of tyrosinase activity. Pigment Cell Res., 11, 12-17.

Pham-Huy, L.A., He, H. and Pham-Huy, C. (2008). Free radicals, antioxidants in disease and health. Int. J. Biomed.Sci., 4, 89-96.

Si, Y.X., Yin, S.J., Park, D., Chung, H.Y., Yan, L. and Lü, Z.R. (2011). Tyrosinase inhibition by isophthalic acid: Kinetics and computational simulation. Int. J. Biol. Macromol., 48, 700-704.

Tanaka, S., Sato, T., Akimoto, N., Yano, M. and Ito, A. (2004). Prevention of UVB-induced photoinflammation and photoaging by a polymethoxy flavonoid, nobiletin, in human keratinocytes in vivo and in vitro. Biochem. Pharmacol., 68, 433-439.

Valero, E., Garcia-Moreno, M., Varon, R. and Garcia-Carmona, F. (1991). Time dependent inhibition of grape polyphenol oxidase by tropolone. J. Agric. Food Chem., 39, 1043-1046.

Xie, L.P., Chen, Q.X., Huang, H., Liu, X.D., Chen, H.T. and Zhang, R.Q. (2003). Inhibitory effects of cupferron on the monophenolase and diphenolase activity of mushroom tyrosinase. Int. J. Bio- 
chem. Cell Biol., 35, 1658-1666.

Yamazaki, Y., Kawano, Y., Yamanaka, A. and Maruyama, S. (2009). $\mathrm{N}-[($ Dihydroxyphenyl)acyl] serotonins as potent inhibitors of tyrosinase from mouse and human melanoma cells. Bioorg. Med. Chem. Lett., 19, 4178-4182.

Yan, Q., Cao, R., Yi, W., Yu, L., Chen, Z., Ma, L. and Song, H. (2009). Synthesis and evaluation of 5-benzylidene(thio)barbiturate-beta-D-glycosides as mushroom tyrosinase inhibitors. Bioorg. Med. Chem. Lett., 19, 4055-4058.

Yoon, J., Fujii, S. and Solomon, E.I. (2009). Geometric and electronic structure differences between the type 3 copper sites of the multicopper oxidases and hemocyanin/tyrosinase. Proc. Natl. Acad. Sci. U.S.A., 106, 6585-6590.

Zhu, Y.J., Song, K.K., Li, Z.C., Pan, Z.Z., Guo, Y.J., Zhou, J.J., Wang, Q., Liu, B. and Chen, Q.X. (2009). Antityrosinase and antimicrobial activities of trans-cinnamaldehyde thiosemicarbazone. J. Agric. Food Chem., 57, 5518-5523.

Zhu, Y.J., Zhou, H.T., Hu, Y.H., Tang, J.Y., Su, M.X. and Guo, Y.J. (2011). Antityrosinase and antimicrobial activities of 2-phenylethanol, 2-phenylacetaldehyde and 2-phenylacetic acid. Food Chem., 124, 298-302. 